\title{
Combined Androgen Blockade in Localized Prostate Cancer Treated With Definitive Radiation Therapy
}

Lucas K. Vitzthum, MDa; Chris Straka, MDa; Reith R. Sarkar, MDa; Rana McKay, MDº; J. Michael Randall, MD;

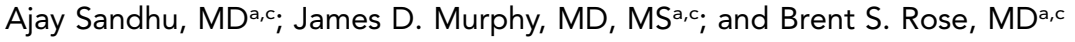

\begin{abstract}
Background: The addition of androgen deprivation therapy to radiation therapy (RT) improves survival in patients with intermediateand high-risk prostate cancer (PCa), but it is not known whether combined androgen blockade (CAB) with a gonadotropin-releasing hormone agonist $(\mathrm{GnRH}-\mathrm{A})$ and a nonsteroidal antiandrogen improves survival over $\mathrm{GnRH}-\mathrm{A}$ monotherapy. Methods: This study evaluated patients with intermediate- and high-risk $\mathrm{PCa}$ diagnosed in 2001 through 2015 who underwent RT with either GnRH-A alone or $\mathrm{CAB}$ using the Veterans Affairs Informatics and Computing Infrastructure. Associations between $C A B$ and prostate cancer-specific mortality (PCSM) and overall survival (OS) were determined using multivariable regression with Fine-Gray and multivariable Cox proportional hazards models, respectively. For a positive control, the effect of long-term versus short-term $\mathrm{GnRH}$-A therapy was tested. Results: The cohort included 8,423 men (GnRH-A, 4,529; CAB, 3,894) with a median follow-up of 5.9 years. There were 1,861 deaths, including 349 resulting from $\mathrm{PCa}$. The unadjusted cumulative incidences of PCSM at 10 years were $5.9 \%$ and $6.9 \%$ for those receiving $\mathrm{GnRH}-\mathrm{A}$ and $C A B$, respectively $(P=.16)$. Compared with $G n R H-A$ alone, $C A B$ was not associated with a significant difference in covariate-adjusted PCSM (subdistribution hazard ratio [SHR], 1.05; 95\% Cl, 0.85-1.30) or OS (hazard ratio, 1.02; $95 \% \mathrm{Cl}, 0.93-1.12$ ). For high-risk patients, long-term versus short-term $\mathrm{GnRH}-\mathrm{A}$ therapy was associated with improved PCSM (SHR, 0.74; 95\% Cl, 0.57-0.95) and OS (SHR, 0.82; $95 \% \mathrm{Cl}, 0.73-0.93)$. Conclusions: In men receiving definitive $\mathrm{RT}$ for intermediate- or high-risk $\mathrm{PCa}, \mathrm{CAB}$ was not associated with improved PCSM or OS compared with $\mathrm{GnRH}$ alone.
\end{abstract}

J Natl Compr Canc Netw 2019;17(12):1497-1504 doi: $10.6004 /$ jnccn.2019.7335

${ }^{a}$ Department of Radiation Medicine and Applied Sciences, ${ }^{b}$ Division of Hematology-Oncology, Department of Internal Medicine, and ${ }^{\mathrm{C} C l i n i c a l}$ and Translational Research Institute, University of California San Diego, San Diego, California.

\section{Background}

Multiple randomized controlled trials have shown that androgen deprivation therapy (ADT) improves outcomes for men with intermediate- or high-risk prostate cancer (PCa) treated with radiation therapy (RT). ${ }^{1-4}$ These trials used combined androgen blockade (CAB) for at least the first 6 months of ADT, and it is not clear whether the addition of an antiandrogen improved outcomes compared with a gonadotropin-releasing hormone agonist (GnRH-A) alone. Accordingly, the optimal ADT regimen remains uncertain. ${ }^{5}$ Of particular interest is whether $\mathrm{CAB}$ with a GnRH-A and a nonsteroidal antiandrogen improves outcomes over GnRH-A monotherapy. ${ }^{6}$

Meta-analyses have shown a modest survival advantage for $\mathrm{CAB}$ over GnRH-A monotherapy in the setting of metastatic PCa. However, CAB was also associated with a marked decrement in quality-of-life domains. ${ }^{7,8}$ No randomized trials examining the effect of adjuvant $\mathrm{CAB}$ versus GnRH-A alone in the setting of definitive RT have been completed. Current practice guidelines note the uncertainty regarding ADT with $\mathrm{CAB}$ versus GnRH-A alone as an optimal first-line therapy in men undergoing definitive RT for PCa. ${ }^{6}$

The purpose of this study was to compare survival outcomes between CAB and GnRH-A monotherapy in men with intermediate- or high-risk PCa treated with definitive RT. We evaluated a cohort of US veterans from the Veterans Affairs (VA) system who were treated in 2001 through 2015 with curative intent. Because there is a risk for residual confounding from selection biases in retrospective studies, we also evaluated the effect on survival of long-term GnRH-A therapy, which has been shown to improve survival in patients with high-risk PCa in prospective randomized control trials, as a positive control., ${ }^{9,10}$

See JNCCN.org for supplemental online content. 


\section{Methods}

\section{Data Source and Patient Selection}

This retrospective cohort study used the VA Informatics and Computing Infrastructure (VINCI), ${ }^{11}$ which contains detailed patient-level data, including demographic information, medical history, medications, procedures, and tumor registry data, gathered according to standardized protocols from the American College of Surgeons. ${ }^{12} \mathrm{~Pa}-$ tients with intermediate- and high-risk PCa treated with definitive external-beam RT and concurrent ADT with a GnRH-A between January 1, 2001, and January 1, 2015, were included (supplemental eFigure 1, available with this article at JNCCN.org). Concurrent ADT was defined as having started a GnRH-A between 6 months before the start of RT and 1 month after. Patients were excluded for positive or unknown nodal or metastatic disease, unknown tumor stage, or up-front chemotherapy. This study was approved by the local VA Institutional Review Board.

\section{Covariates, Exposures, and Outcomes}

Patient demographic information, cancer stage according to the 7th edition of the AJCC Cancer Staging Manual, Gleason score, and pretreatment prostate-specific antigen (PSA) were obtained from the tumor registry (Table 1). Intermediate-risk disease was defined by stage $\mathrm{T} 2 \mathrm{~b}-\mathrm{c}$ disease, Gleason score 7, or PSA level of 10 to $20 \mathrm{ng} / \mathrm{mL}$. ${ }^{13,14}$ High-risk disease was defined by stage $\geq \mathrm{T} 3$ disease, Gleason score $\geq 8$, or PSA level $\geq 20 \mathrm{ng} / \mathrm{mL} .{ }^{13,14}$ CharlsonDeyo comorbidity index score was determined using ICD-9/10 diagnostic codes from the year before PCa diagnosis as previously described. ${ }^{15,16}$

Information on treatment with $\mathrm{ADT}$ was obtained from the VA pharmacy database. CAB was defined as treatment with a nonsteroidal androgen receptor antagonist (eg, bicalutamide) started within 1 month of GnRH-A therapy for any duration. Some patients are treated with a short course of antiandrogen with the intent to counteract the temporary surge in testosterone seen with a GnRH-A. To test the sensitivity of antiandrogen therapy duration, patients who received $C A B$ treatment were further classified as having received long-course ( $>30$ days) versus short-course ( $\leq 30$ days) antiandrogen based on prescription fill data. Overall survival (OS) was obtained through tumor registry data. For prostate cancer-specific mortality (PCSM), cause of death was determined from the National Death Index as previously described. ${ }^{17}$

\section{Statistical Methods}

Baseline covariates were compared between the treatment arms using the Student $t$ or chi-square test. Data were missing for tobacco use $(7.7 \%)$, median income (2.9\%), percentage with a high school diploma by ZIP

\begin{tabular}{|c|c|c|c|}
\hline & GnRH-A & CAB & $P$ Value \\
\hline Patients, N & 4,529 & 3,894 & \\
\hline Age, y, mean (SD) & $66.25(6.96)$ & $66.62(7.49)$ & .02 \\
\hline Race, n (\%) & & & $<.001$ \\
\hline White & $2,950(65.1)$ & $2,450(62.9)$ & \\
\hline Black & $1,432(31.6)$ & $1,360(34.9)$ & \\
\hline Other & $147(3.2)$ & $84(2.2)$ & \\
\hline Body mass index, n (\%) & & & .316 \\
\hline Healthy weight & $1,046(23.1)$ & $953(24.5)$ & \\
\hline Overweight & $3,357(74.1)$ & $2,830(72.7)$ & \\
\hline Underweight & $126(2.8)$ & $111(2.9)$ & \\
\hline $\mathrm{CCl}$ score, n (\%) & & & .669 \\
\hline 0 & $3,183(70.3)$ & $2,763(71.0)$ & \\
\hline 1 & $800(17.7)$ & $659(16.9)$ & \\
\hline$\geq 2$ & $546(12.1)$ & $472(12.1)$ & \\
\hline \multicolumn{4}{|l|}{ ZIP code metrics } \\
\hline $\begin{array}{l}\text { Median income per } \\
\$ 1,000 \text { USD, mean (SD) }\end{array}$ & $48.1(17.3)$ & $49.9(19.7)$ & $<.001$ \\
\hline $\begin{array}{l}\text { Percentage with high } \\
\text { school diploma, mean (SD) }\end{array}$ & $0.85(0.08)$ & $0.85(0.09)$ & .001 \\
\hline Married, n (\%) & $2,338(51.6)$ & $1,848(47.5)$ & $<.001$ \\
\hline Tobacco use, n (\%) & & & .586 \\
\hline Current & $1,524(36.6)$ & $1,301(36.3)$ & \\
\hline None & 994 (23.9) & $892(24.9)$ & \\
\hline Former & $1,643(39.5)$ & $1,391(38.8)$ & \\
\hline Employed & 705 (15.6) & $591(15.2)$ & .643 \\
\hline T stage, n (\%) & & & $<.001$ \\
\hline 1 & $2,707(59.8)$ & $2,166(55.6)$ & \\
\hline 2 & $1,654(36.5)$ & $1,497(38.4)$ & \\
\hline 3 & $155(3.4)$ & $209(5.4)$ & \\
\hline 4 & $13(0.3)$ & $22(0.6)$ & \\
\hline Gleason score, n (\%) & & & $<.001$ \\
\hline 6 & $283(6.2)$ & $232(6.0)$ & \\
\hline 7 & $2,517(55.6)$ & $1,897(48.7)$ & \\
\hline 8 & $1,021(22.5)$ & $1,029(26.4)$ & \\
\hline 9 & $653(14.4)$ & $649(16.7)$ & \\
\hline 10 & $55(1.2)$ & $87(2.2)$ & \\
\hline PSA, ng/mL, mean (SD) & $12.30(12.53)$ & $13.97(14.17)$ & $<.001$ \\
\hline High risk, n (\%) & $2,145(47.4)$ & $2,242(57.6)$ & $<.001$ \\
\hline
\end{tabular}

Abbreviations: $\mathrm{CAB}$, combined androgen blockade; $\mathrm{CCl}$, Charlson-Deyo comorbidity index; GnRH-A, gonadotropin-releasing hormone agonist; PSA, prostate-specific antigen.

code $(2.0 \%)$, and body mass index category $(0.2 \%)$. Missing data were imputed for survival analysis using multivariate imputation by chained equations. ${ }^{18}$

Unadjusted survival was compared between the treatment groups using the log-rank test for OS and 
cumulative incidence curves and univariable Fine-Gray regression for PCSM. ${ }^{19}$ Multivariable Cox proportional hazards and Fine-Gray models were fit to determine the covariate-adjusted hazard of $\mathrm{CAB}$ in patients with high- and intermediate-risk PCa for OS and PCSM, respectively. Covariates were selected using backward selection and included with a $P$ value $<.2$. Survival analyses were stratified by high- and intermediate-risk disease because ADT has been shown to have differing effects between these groups. ${ }^{4}$ An additional multivariable analysis was performed comparing patients treated with GnRH-A alone versus long-course (>30 days) antiandrogen to determine whether a longer antiandrogen course was needed for a therapeutic effect.

As a positive control, we evaluated the adjusted hazard for long-term ( $\geq 1$ year) versus short-term $(<1$ year) ADT, which has been shown to improve survival in men with localized high-risk PCa in prospective randomized trials. ${ }^{9,10}$ Covariate-adjusted survival curves were plotted for OS using a conditional analysis method to balance subgroups with respect to confounding variables. ${ }^{20}$ Statistical analysis and modeling were performed using $\mathrm{R}$ version 3.5.1 (R Foundation for Statistical Computing).

\section{Results}

\section{Patient Characteristics}

The cohort comprised 8,423 patients who were treated with a GnRH-A alone $(n=4,529 ; 53.8 \%)$ or $\mathrm{CAB}(\mathrm{n}=3,894$; $46.2 \%$ ) (supplemental eFigure 1). Among those treated with $\mathrm{CAB}, 47.3 \%(n=1,842)$ were treated with long-course ( $>30$ days) antiandrogen therapy. The median length of GnRH-A therapy was 8.6 months for the entire cohort, and median durations of ADT for men with intermediateand high-risk PCa were 6.0 and 15.6 months, respectively.
Patient and demographic factors were similar, with no substantial differences in age, body mass index, or Charlson-Deyo comorbidity index score between the GnRH-A and CAB treatment groups. Patients treated with $\mathrm{CAB}$ lived in ZIP codes with a slightly higher median income ( $\$ 49,900$ vs $\$ 48,100 ; P<.001$ ) but were less likely to be married $(47.5 \%$ vs $51.6 \% ; P<.001)$. The $\mathrm{CAB}$ group was more likely to have clinical stages $\mathrm{T} 3-4$ disease $(6.0 \%$ vs $3.7 \%$; $P<.001)$, Gleason score $\geq 8$ (45.3\% vs $38.1 \% ; P<.001$ ), high-risk classification ( $57.6 \%$ vs $47.4 \%$; $P<.001)$, and higher mean pretreatment PSA value (13.97 vs $12.30 \mathrm{ng} / \mathrm{mL} ; P<.001$ ).

\section{Survival Outcomes}

Median follow-up was 5.9 years. A total of 1,861 deaths of any cause occurred, including 349 deaths resulting from PCa. There was no difference in the cumulative incidence of PCSM for those receiving GnRH-A versus CAB (10-year cumulative incidence, $5.9 \%$ vs $6.9 \%$, respectively; $P=.14$ ) (Figure 1). Similarly, there was no difference in PCSM between GnRH-A alone and any CAB when stratified by high-risk (10-year cumulative incidence, $8.4 \%$ vs $8.4 \% ; P=.82$ ) and intermediate-risk groups (10-year cumulative incidence, $3.8 \%$ vs $5.0 \% ; P=.39$ ).

\section{Multivariable Regression}

Compared with GnRH-A monotherapy, $\mathrm{CAB}$ was not associated with a significant difference in covariate-adjusted PCSM (subdistribution hazard ratio [SHR], 1.05; 95\% CI, $0.85-1.30 ; P=.63$ ) or OS (hazard ratio [HR], 1.02; 95\% CI, $0.93-1.12 ; P=.65$ ) (Table 2 ). When separated by risk group, no difference in PCSM was seen among those treated with $\mathrm{CAB}$ versus GnRH-A monotherapy for either high-risk (SHR, 1.00; 95\% CI, 0.77-1.29; $P=.98$ ) or intermediaterisk patients (SHR, 1.19; 95\% CI, 0.81-1.75; $P=.37$ ).
A
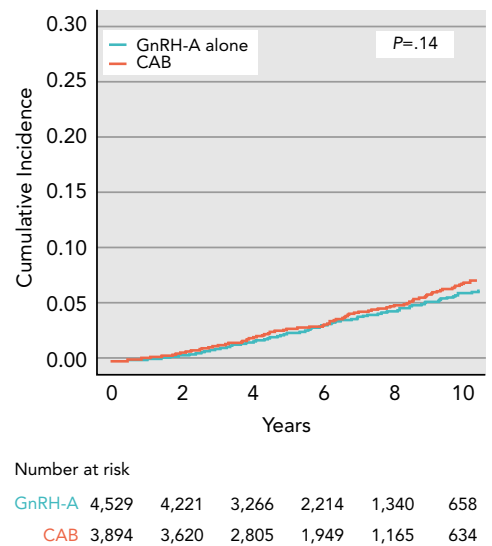

B
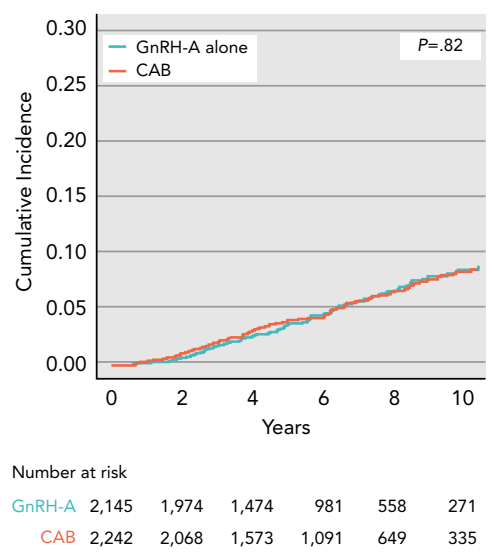

C

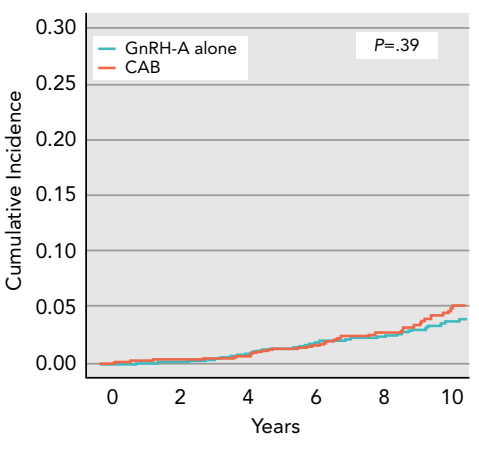

Number at risk

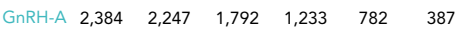
$\begin{array}{lllllll}\text { CAB } & 1,652 & 1,552 & 1,232 & 858 & 516 & 299\end{array}$

Figure 1. Cumulative incidence of prostate cancer mortality for patients treated with a GnRH-A versus $C A B$ among (A) the entire cohort, (B) high-risk patients, and (C) intermediate-risk patients. $P$ values were derived from univariable Fine-Gray model.

Abbreviations: $\mathrm{CAB}$, combined androgen blockade; GnRH-A, gonadotropin-releasing hormone agonist. 
Table 2. Multivariable Regression for OS and PCSM

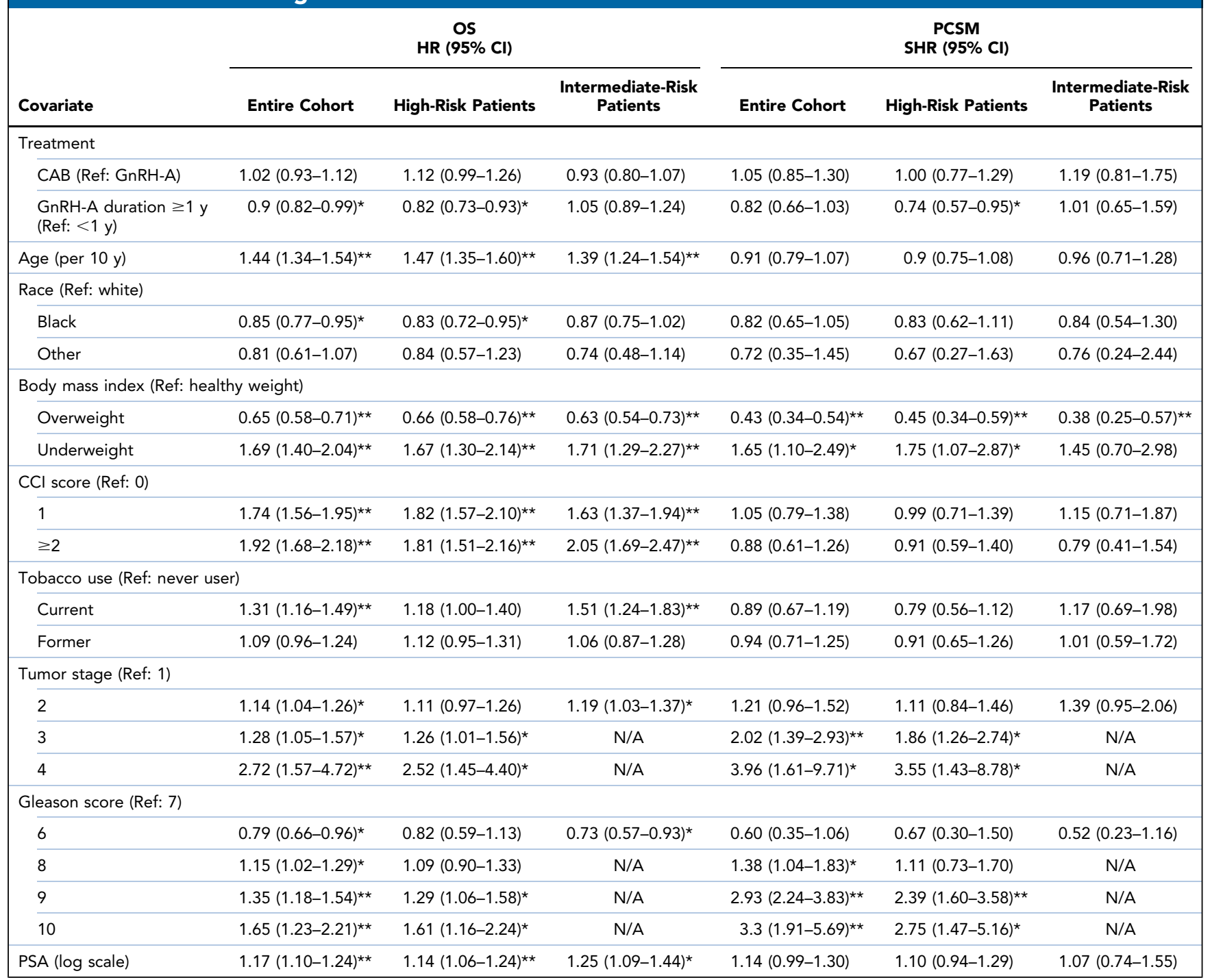

Abbreviations: $\mathrm{CCl}$, Charlson-Deyo comorbidity index; $\mathrm{CAB}$, combined androgen blockade; GnRH-A, gonadotropin-releasing hormone agonist; HR, hazard ratio; N/A, not applicable; OS, overall survival; PCSM, prostate cancer-specific mortality; PSA, prostate-specific antigen; SHR, subdistribution hazard ratio. ${ }^{\star} P<.05$.

$\star \star P<.001$

Similarly, CAB was not associated with increased OS among high-risk (HR, 1.12; 95\% CI, 0.99-1.26; $P=.08$ ) or intermediate-risk patients (HR, 0.93; 95\% CI, 0.80-1.07; $P=.29$ ) (Figure 2).

In a sensitivity analysis excluding patients treated with an antiandrogen for $<30$ days, there was still no difference in covariate-adjusted PCSM between patients treated with $\mathrm{CAB}$ and those who received GnRH-A monotherapy among the entire cohort (SHR, 1.22; 95\% CI, 0.95-1.85; $P=.24$ ), high-risk disease (SHR, 1.26; 95\% CI, 0.94-1.69; $P=.12$ ), or intermediate-risk disease (HR, 1.2; 95\% CI, 0.74-2.1; $P=.50$ ). Likewise, no improvement was seen in covariate-adjusted OS with long-term antiandrogen over GnRH-A monotherapy among the entire cohort
(HR, 0.99; 95\% CI, 0.88-1.12; $P=.91$ ), high-risk patients (HR, 1.1; 95\% CI, 0.95-1.28; $P=.22$ ), or intermediate-risk patients (HR, 1.2; 95\% CI, 0.73-1.99; $P=.22$ ).

Covariates associated with an increased risk for OS and PCSM included advanced tumor stage (T3 or T4), high Gleason score (9 or 10), and underweight BMI category (Table 2). Advanced age and presence of comorbidity were associated with an increased hazard for OS but not PCSM. Having a higher PSA level was associated with worse OS among both high- and intermediate-risk patients.

\section{Duration of ADT}

Patients treated with ADT for $\geq 1$ year were significantly more likely to have stage T3-4 disease ( $7.6 \%$ vs $2.8 \%$; 
A

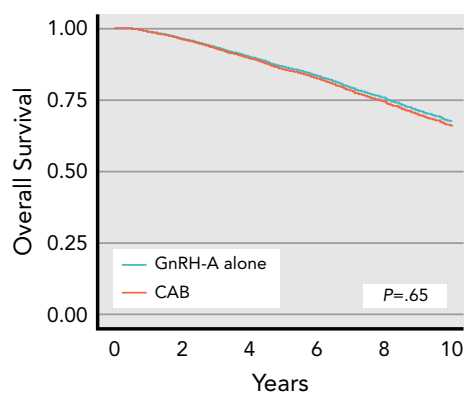

B

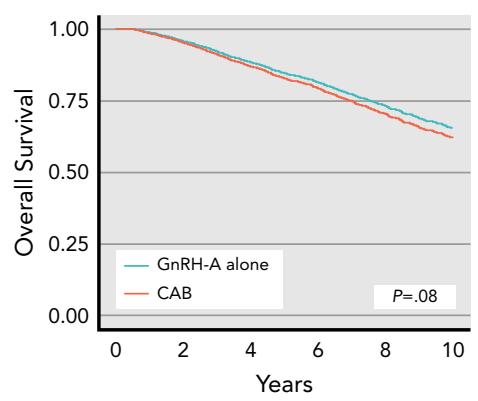

C

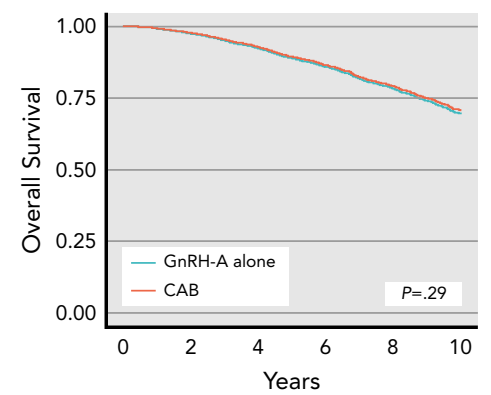

Figure 2. Adjusted overall survival for patients treated with a GnRH-A versus $C A B$ among $(A)$ the entire cohort, $(B)$ high-risk patients, and (C) intermediate-risk patients. $P$ values were derived from multivariable Cox proportional hazards model.

Abbreviations: $\mathrm{CAB}$, combined androgen blockade; $\mathrm{GnRH}-\mathrm{A}$, gonadotropin-releasing hormone agonist.

$P<.001)$, Gleason score $\geq 8(59.2 \%$ vs $27.1 \% ; P<.001)$, and high-risk classification $(76.6 \%$ vs $36.1 \% ; P<.001)$. No significant difference was seen in the unadjusted cumulative incidence of PCSM between patients treated with long-term versus short-term $\mathrm{ADT}$ for the entire cohort (10-year cumulative incidence, $6.5 \%$ vs $6.3 \%$; $P=.18$ ), highrisk patients (10-year cumulative incidence, $7.5 \%$ vs $9.7 \%$; $P=.06$ ), or intermediate-risk patients (10-year cumulative incidence, $3.9 \%$ vs $4.5 \%$; $P=.79$ ).

When the analysis was controlled for confounding factors in the multivariable Fine-Gray model, long-term ADT was associated with improved PCSM compared with short-term ADT in high-risk patients (SHR, 0.74; 95\% CI, $0.57-0.95 ; P=.02$ ) but not intermediate-risk patients (SHR, 1.01; 95\% CI, $0.65-1.59 ; P=.94$ ) or the combined cohort (SHR, 0.82; 95\% CI, 0.66-1.03; $P=.09$ ) (Table 2). Long-term ADT was also associated with improved covariate-adjusted OS among the entire cohort (HR, 0.90; 95\% CI, 0.82-0.99; $P=.04$ ) and high-risk patients (HR, 0.82; 95\% CI, 0.73-0.93; $P=.002$ ) but not intermediate-risk patients (HR, 1.05; 95\% CI, 0.89-1.24; $P=.59$ ) (Figure 3, Table 2).

\section{Discussion}

In a large population-based cohort of patients with intermediate- and high-risk PCa treated with definitive RT, we found that $\mathrm{CAB}$ was not significantly associated with improved PCSM or OS compared with GnRH-A alone when controlling for confounding factors. This finding was consistent across both high- and intermediate-risk groups and when evaluating duration of antiandrogen use. In contrast, long-term GnRH therapy was associated with improved OS and reduced PCSM in patients with high-risk disease, which is consistent with findings of prior prospective randomized trials. ${ }^{9,10}$

There are 3 main hypothetical reasons to use an antiandrogen with GnRH-A. First, GnRH-A alone leads to a transient increase in testosterone levels, sometimes called a testosterone flare, before testosterone decreases to castrate levels. In certain patients, such as those with substantial urinary outflow obstructive symptoms, blocking the testosterone flare may reduce the risk of urinary retention with a GnRH-A. Second, GnRH-A alone does not suppress extratesticular sources of androgens, such as the adrenal glands and the tumor itself. ${ }^{21-23}$ Third, several
A

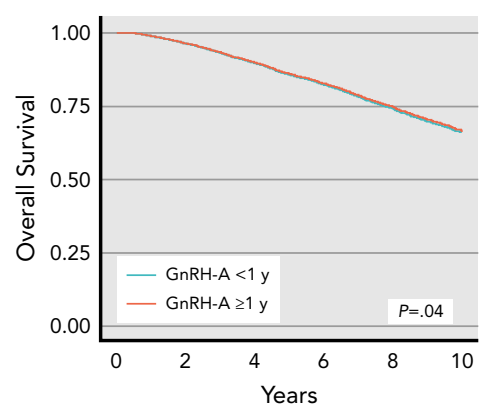

B

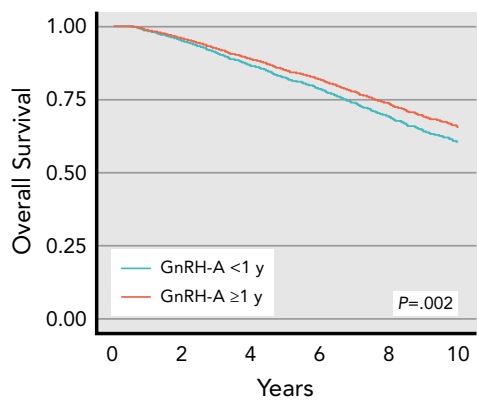

C

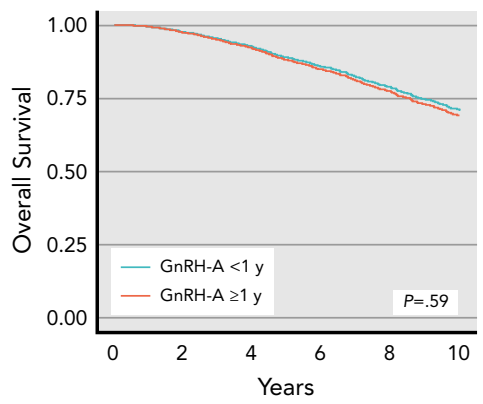

Figure 3. Adjusted overall survival for patients treated with a GnRH-A for $<1$ versus $\geq 1$ year among (A) the entire cohort, (B) high-risk patients, and $(C)$ intermediate-risk patients. $P$ values were derived from multivariable Cox proportional hazards model.

Abbreviation: $\mathrm{GnRH}-\mathrm{A}$, gonadotropin-releasing hormone agonist. 
studies have shown that despite castrate serum levels of testosterone $<50 \mathrm{ng} / \mathrm{mL}$ with $\mathrm{GnRH}-\mathrm{A}$ alone, tissue androgen levels can persist at $25 \%$ to $35 \%$ of serum levels. ${ }^{24}$ Persistent expression of androgen receptorregulated genes can be seen in prostate tissue despite castration, suggesting that more intense androgen suppression may be warranted. ${ }^{11}$

Nonsteroidal antiandrogens act directly on the androgen receptor and competitively inhibit ligand binding, potentially ameliorating the effects of residual testosterone and/or small molecule activation seen in treatment with GnRH-A alone. However, the binding affinity of first-generation antiandrogens for the androgen receptor is relatively low compared with newer agents such as enzalutamide, which also inhibits nuclear translocation of the androgen receptor, DNA binding, and coactivator recruitment. ${ }^{25,26}$ Therefore, the impact of adding antiandrogen to GnRH-A may not be sufficient to meaningfully suppress androgen signaling in PCa cells beyond what is achieved with GnRH-A alone.

In the 1990s and early 2000s, several randomized trials showed a small survival advantage to $\mathrm{CAB}$ compared with GnRH-A alone in the setting of metastatic PCa. ${ }^{27,28} \mathrm{~A}$ systematic review showed that $\mathrm{CAB}$ use in metastatic PCa was associated with a small survival advantage $(27.6 \%$ vs $24.7 \%$; $P=.005)$ compared with GnRH-A alone at 5 years, but also showed a marked decrement in quality-of-life domains with $\mathrm{CAB}$, and thus recommended GnRH-A alone for most patients. ${ }^{7}$ A meta-analysis of $\mathrm{CAB}$ versus GnRH-A alone found a higher rate of treatment withdrawal $(10 \%$ vs $2 \%)$ and worse emotional functioning with $\mathrm{CAB} .{ }^{8}$ Current practice guidelines list GnRH agonist/antagonist monotherapy or $\mathrm{CAB}$ as options for patients with PCa with metastatic disease, but they reference multiple studies advocating for monotherapy over $\mathrm{CAB}$ to minimize adverse effects. ${ }^{6,29,30}$

Considering the added adverse effects of $\mathrm{CAB}$ and limited efficacy in the metastatic setting, the role of $\mathrm{CAB}$ compared with GnRH-A alone with RT is not clear, and current practice guidelines note the uncertainty regarding $\mathrm{ADT}$ with $\mathrm{CAB}$ versus $\mathrm{GnRH}-\mathrm{A}$ alone as an optimal first-line therapy in men undergoing definitive RT for PCa. ${ }^{6}$ Approximately half of the patients in our cohort received $\mathrm{CAB}$ and half received GnRH-A monotherapy, suggesting a lack of consensus in current clinical practice. In this analysis, when controlling for patient and cancer factors, we did not observe a disease-specific survival or OS advantage associated with short- or long-term $\mathrm{CAB}$ versus GnRH monotherapy among the entire cohort, intermediate-risk patients, or high-risk patients. Our analysis did show more aggressive disease characteristics in patients receiving $C A B$ compared with GnRH-A alone. Patients who received hormone therapy for $>1$ year also had more aggressive disease, but high-risk patients in this group were found to have improved OS and PCSM in adjusted analysis, consistent with results of prior randomized trials. ${ }^{3,4,31}$

Limited published data are available comparing $\mathrm{CAB}$ versus GnRH-A alone in patients treated with definitive RT. ${ }^{32,33}$ One study published by Nanda et $\mathrm{al}^{32}$ in 2010 found an improved PCSM in multivariable analysis. However, there are several important considerations. First, the study was relatively small, with only 13 deaths resulting from PCa; in contrast, 349 deaths resulting from PCa were reported in our study, allowing a more robust multivariable model. In addition, despite all patients having high-risk disease in the study by Nanda et $\mathrm{al}^{32}$ the median duration of ADT was only 4.3 months, consistent with the earlier time period of that study. The median duration of ADT for men with high-risk PCa in our study was 15.6 months. Multiple subsequent publications have shown a benefit of long-term ( $>1$ year) ADT in this population, $, 3,31,34$ and it is possible that a longer duration of GnRH-A alone makes up for the omission of an antiandrogen during the first few weeks or months of therapy. Finally, all patients in the study by Nanda et $\mathrm{al}^{32}$ received brachytherapy as a component of their RT, which may limit the generalizability of these findings to patients receiving external-beam RT. Another study, involving 102 men with PCa with an unfavorable risk profile enrolled in a clinical trial, compared patients treated with $\mathrm{CAB}$ for 6 months versus those who discontinued the antiandrogen therapy early. ${ }^{33}$ In multivariable analysis, a longer duration of antiandrogen therapy was associated with a decreased risk of biochemical failure. Again, the longer median duration of ADT in our cohort (15.6 vs 6 months) could explain the discordant results between these 2 studies.

This study has potential limitations. Most notably, given its retrospective nature, there may be residual confounding from unmeasured biases between treatment groups. In addition, tumor registry data did not contain the predominant Gleason pattern or percentage of positive cores, and thus we were unable to separate favorable and unfavorable intermediate-risk patients in our analysis. The addition of favorable intermediate-risk patients could conceivably have decreased our power to detect an advantage to treatment with $\mathrm{CAB}$ in the intermediate-risk population, although it seems unlikely that this population would benefit from $\mathrm{CAB}$ when the high-risk population did not. This study is also limited by a lack of toxicity data, although prior randomized trials in the metastatic setting have shown a decrease in quality of life with CAB. ${ }^{7,8}$ Outcomes relating to biochemical failure and progression of distant metastases are also of interest but are not available in this dataset. 
Finally, in the past decade, more potent drugs have been developed to limit the effect of testosterone on PCa. Enzalutamide is a second-generation androgen receptor inhibitor that has been shown to confer a survival advantage in the setting of metastatic PCa. ${ }^{35,36}$ Abiraterone blocks androgen synthesis at all body sites and has been shown in randomized trials to confer a survival advantage in metastatic castration-resistant PCa before and after docetaxel and also in castration-sensitive PCa before docetaxel. ${ }^{37-39}$ More recently, early results of the STAMPEDE trial showed an OS advantage to treatment with ADT plus abiraterone versus ADT alone in a mixed cohort of patients with locally advanced and metastatic castrationsensitive PCa, and a subset analysis showed a large failure-free survival advantage in patients with locally advanced or node-positive disease receiving definitiveintent therapy. ${ }^{40}$

\section{Conclusions}

The addition of a first-generation antiandrogen to a GnRH-A was not associated with any improvement in
OS or PCSM when administered with definitive RT to patients with intermediate- and high-risk PCa. These data suggest that there is a need for further research to determine the optimal ADT regimen for men treated with definitive RT.

\section{Submitted April 12, 2019; accepted for publication June 19, 2019.}

Author contributions: Study concept: Vitzthum, Straka, McKay, Sandhu, Rose. Data analysis: Vitzthum, Straka, Sarkar, Murphy, Rose. Data curation: Sarkar. Writing of manuscript: Vitzthum, Straka, McKay, Randall, Sandhu, Murphy, Rose. Manuscript editing: Vitzthum, Straka, Randall, Rose.

Disclosures: Dr. McKay has disclosed that he is a scientific advisor for Janssen. The remaining authors have disclosed that they have not received any financial considerations from any person or organization to support the preparation, analysis, results, or discussion of this article.

Funding: This work was supported by NIH grant TL1TR001443 (Sarkar).

Disclaimer: The content is solely the responsibility of the authors and does not necessarily represent the official views of the $\mathrm{NIH}$.

Correspondence: Brent S. Rose, MD, Department of Radiation Medicine and Applied Sciences, University of California San Diego, Altman Clinical and Translational Research Institute Building, 9452 Medical Center Drive, La Jolla, CA 92037. Email: bsrose@ucsd.edu

\section{References}

1. Jones CU, Hunt D, McGowan DG, et al. Radiotherapy and short-term androgen deprivation for localized prostate cancer. N Engl J Med 2011;365:107-118

2. D'Amico AV, Chen $M H$, Renshaw $A A$, et al. Androgen suppression and radiation vs radiation alone for prostate cancer: a randomized trial. JAMA 2008;299:289-295.

3. Bolla M, de Reijke TM, Van Tienhoven G, et al. Duration of androgen suppression in the treatment of prostate cancer. N Engl J Med 2009; 360:2516-2527.

4. Horwitz EM, Bae K, Hanks GE, et al. Ten-year follow-up of Radiation Therapy Oncology Group protocol 92-02: a phase III trial of the duration of elective androgen deprivation in locally advanced prostate cancer. J Clin Oncol 2008;26:2497-2504.

5. Sharifi N, Gulley JL, Dahut WL. Androgen deprivation therapy for prostate cancer. JAMA 2005;294:238-244.

6. Mohler JL, Antonarakis ES, Armstrong AJ, et al. NCCN Clinical Practice Guidelines in Oncology: Prostate Cancer. Version 2.2019. To view the most recent version, visit NCCN.org. Accessed April 15, 2019.

7. Lukka $\mathrm{H}$, Waldron $\mathrm{T}, \mathrm{Klotz} \mathrm{L}$, et al. Maximal androgen blockade for the treatment of metastatic prostate cancer - a systematic review. Curr Oncol 2006;13:81-93.

8. Samson DJ, Seidenfeld J, Schmitt B, et al. Systematic review and meta-analysis of monotherapy compared with combined androgen blockade for patients with advanced prostate carcinoma. Cancer 2002 95:361-376.

9. Zapatero A, Guerrero A, Maldonado X, et al. High-dose radiotherapy with short-term or long-term androgen deprivation in localised prostate cancer (DART01/05 GICOR): a randomised, controlled, phase 3 trial. Lancet Oncol 2015;16:320-327.

10. Hanks GE, Pajak TF, Porter A, et al. Phase III trial of long-term adjuvant androgen deprivation after neoadjuvant hormonal cytoreduction and radiotherapy in locally advanced carcinoma of the prostate: the Radiation Therapy Oncology Group protocol 92-02. J Clin Oncol 2003;21: 3972-3978.

11. Mostaghel EA, Nelson PS, Lange $P$, et al. Targeted androgen pathway suppression in localized prostate cancer: a pilot study. J Clin Oncol 2014; 32:229-237.

12. Commission on Cancer. FORDS: Facility Oncology Registry Data Standards: Revised for 2016. Available at: https://www.facs.org/ /media/ files/quality\%20programs/cancer/ncdb/fords\%202016.as. Accessed October 10, 2019.
13. D'Amico AV, Whittington R, Malkowicz SB, et al. Biochemical outcome after radical prostatectomy, external beam radiation therapy, or interstitial radiation therapy for clinically localized prostate cancer. JAMA 1998;280:969-974.

14. Mohler JL, Antonarakis ES, Armstrong AJ, et al. NCCN Clinical Practice Guidelines in Oncology: Prostate Cancer. Version 2.2019. J Natl Compr Canc Netw 2019;17:479-505.

15. Charlson ME, Pompei $\mathrm{P}$, Ales $\mathrm{KL}$, et al. A new method of classifying prognostic comorbidity in longitudinal studies: development and validation. J Chronic Dis 1987;40:373-383.

16. Bryant AK, Huynh-Le MP, Simpson DR, et al. Association of HIV status with outcomes of anal squamous cell carcinoma in the era of highly active antiretroviral therapy. JAMA Oncol 2018;4:120-122.

17. Bryant AK, McKay RR, Kader AK, et al. Subcastrate testosterone nadir and clinical outcomes in intermediate- or high-risk localized prostate cancer. Int J Radiat Oncol Biol Phys 2019;103:1068-1076.

18. Buuren S, Groothuis-Oudshoorn K. Multivariate imputation by chained equations in R. J Stat Softw 2011;45:1-67.

19. Fine JP, Gray RJ. A proportional hazards model for the subdistribution of a competing risk. J Am Stat Assoc 1999;94:496-509.

20. Nieto FJ, Coresh J. Adjusting survival curves for confounders: a review and a new method. Am J Epidemiol 1996;143:1059-1068.

21. Mostaghel EA, Page ST, Lin DW, et al. Intraprostatic androgens and androgen-regulated gene expression persist after testosterone suppression: therapeutic implications for castration-resistant prostate cancer Cancer Res 2007;67:5033-5041.

22. Schmitt B, Bennett C, Seidenfeld J, et al. Maximal androgen blockade for advanced prostate cancer. Cochrane Database Syst Rev 2000 CD001526.

23. Labrie F, Veilleux R, Fournier A. Low androgen levels induce the development of androgen-hypersensitive cell clones in Shionogi mouse mammary carcinoma cells in culture. J Natl Cancer Inst 1988; 80:1138-1147.

24. Oefelein MG, Feng A, Scolieri MJ, et al. Reassessment of the definition of castrate levels of testosterone: implications for clinical decision making. Urology 2000;56:1021-1024.

25. Uemura $\mathrm{H}$. New generation of androgen receptor antagonist in castration resistant prostate cancer [in Japanese]. Nihon Rinsho 2014; 72:2164-2169.

26. Siemens DR, Klotz L, Heidenreich A, et al. Efficacy and safety of enzalutamide vs bicalutamide in younger and older patients with 
metastatic castration resistant prostate cancer in the TERRAIN trial. J Urol 2018;199:147-154.

27. Denis L, Murphy GP. Overview of phase III trials on combined androgen treatment in patients with metastatic prostate cancer. Cancer 1993; 72(12 Suppl):3888-3895.

28. Klotz L. Maximal androgen blockade for advanced prostate cancer. Best Pract Res Clin Endocrinol Metab 2008;22:331-340.

29. Laufer M, Denmeade SR, Sinibaldi VJ, et al. Complete androgen blockade for prostate cancer: what went wrong? J Urol 2000;164:3-9.

30. Loblaw DA, Virgo KS, Nam R, et al. Initial hormonal management of androgen-sensitive metastatic, recurrent, or progressive prostate cancer: 2006 update of an American Society of Clinical Oncology practice guideline. J Clin Oncol 2007;25:1596-1605.

31. Zapatero A, Guerrero A, Maldonado X, et al. High-dose radiotherapy with short-term or long-term androgen deprivation in localised prostate cancer (DART01/05 GICOR): a randomised, controlled, phase 3 trial. Lancet Oncol 2015;16:320-327.

32. Nanda A, Chen MH, Moran BJ, et al. Total androgen blockade versus a luteinizing hormone-releasing hormone agonist alone in men with high-risk prostate cancer treated with radiotherapy. Int J Radiat Oncol Biol Phys 2010;76:1439-1444.
33. D'Amico AV, Chen $\mathrm{MH}$, Renshaw $\mathrm{AA}$, et al. Risk of prostate cancer recurrence in men treated with radiation alone or in conjunction with combined or less than combined androgen suppression therapy. J Clin Oncol 2008;26:2979-2983.

34. Feng FY, Blas K, Olson $\mathrm{K}$, et al. Retrospective evaluation reveals that long term androgen deprivation therapy improves cause-specific and overall survival in the setting of dose-escalated radiation for high-risk prostate cancer. Int J Radiat Oncol Biol Phys 2013;86:64-71.

35. Beer TM, Armstrong AJ, Rathkopf DE, et al. Enzalutamide in metastatic prostate cancer before chemotherapy. N Engl J Med 2014;371:424-433.

36. Scher HI, Fizazi K, Saad F, et al. Increased survival with enzalutamide in prostate cancer after chemotherapy. N Engl J Med 2012;367:1187-1197.

37. de Bono JS, Logothetis CJ, Molina A, et al. Abiraterone and increased survival in metastatic prostate cancer. N Engl J Med 2011;364:1995-2005.

38. Fizazi K, Tran N, Fein L, et al. Abiraterone plus prednisone in metastatic, castration-sensitive prostate cancer. N Engl J Med 2017;377:352-360.

39. Ryan CJ, Smith MR, de Bono JS, et al. Abiraterone in metastatic prostate cancer without previous chemotherapy. N Engl J Med 2013;368:138-148.

40. James ND, de Bono JS, Spears MR, et al. Abiraterone for prostate cancer not previously treated with hormone therapy. N Engl J Med 2017; $377: 338-351$.

\section{See JNCCN.org for supplemental online content.}


Supplemental online content for:

\section{Combined Androgen Blockade in Localized Prostate Cancer Treated With Definitive Radiation Therapy}

Lucas K. Vitzthum, MD; Chris Straka, MD; Reith R. Sarkar, MD; Rana McKay, MD; J. Michael Randall, MD; Ajay Sandhu, MD; James D. Murphy, MD, MS; and Brent S. Rose, MD

J Natl Compr Canc Netw 2019;17(12):1497-1504

eFigure 1: Cohort Inclusion and Exclusion Criteria 


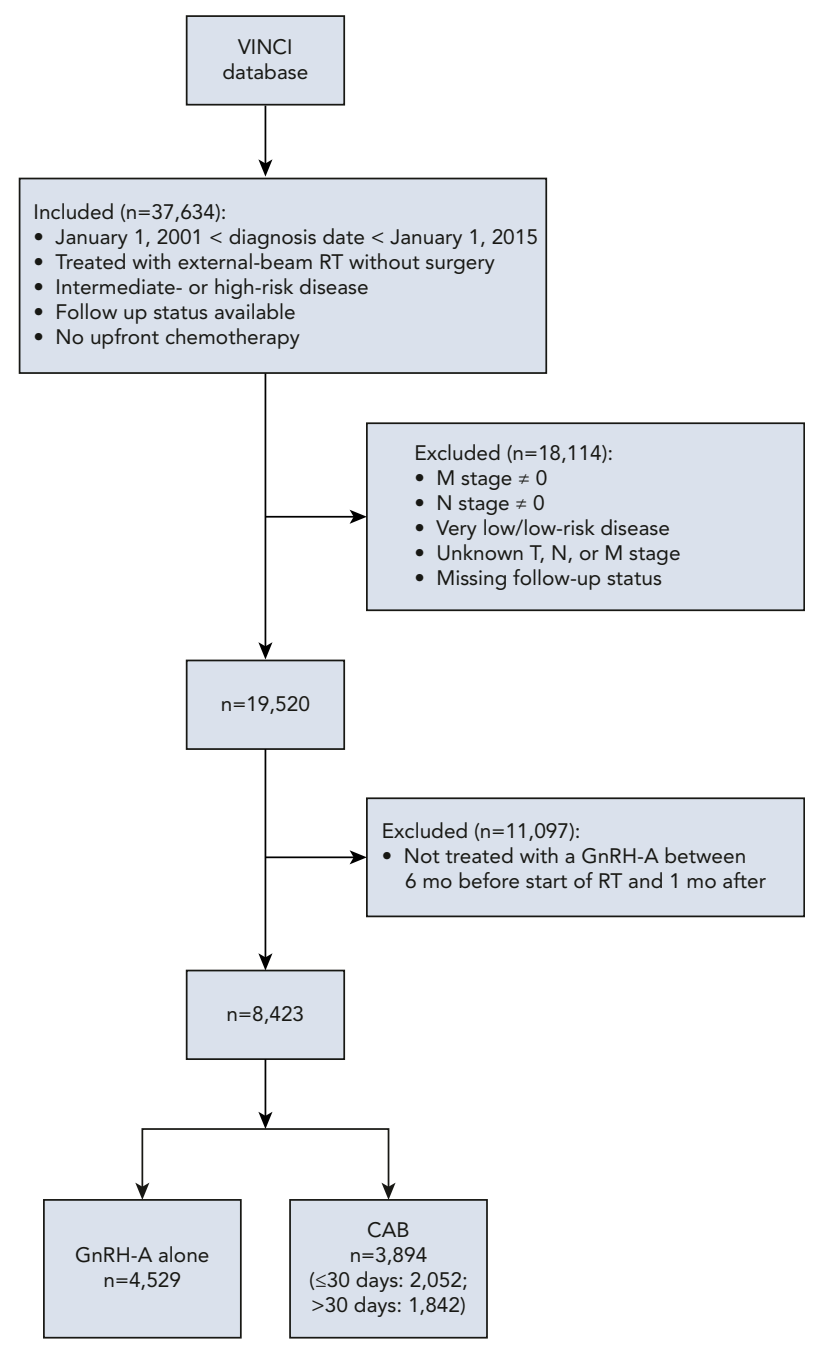

eFigure 1. Cohort inclusion and exclusion criteria.

Abbreviations: $\mathrm{CAB}$, combined androgen blockade; GnRH-A, gonadotropin-releasing hormone agonist; RT, radiation therapy; VINCl, Veterans Affairs Informatics and Computing Infrastructure. 